\title{
RAYLEIGH WAVES AND FREE SURFACE REFLECTIONS*
}

BY

C. H. DIX, C. Y. FU, AND ETHEL W. MCLEMORE United Geophysical Company, Pasadena, California

1. Introduction. The theory of waves associated with the plane boundary of a semi-infinite, isotropic, homogeneous, perfectly elastic medium was first given by Lord Rayleigh, ${ }^{1}$ who discussed the problem for plane waves of fixed frequency. Many papers have been written giving treatments of variations of the problem studied by Rayleigh but the treatment in Rayleigh's original paper contained most of the results of interest for plane waves.

Had Lord Rayleigh realized the great practical importance of his surface waves, he would doubtless have included more numerical results in his original paper, and the material of the present paper would have been more or less completely included therein. Rayleigh waves are important in the seismic method of oil exploration since they generally occur as a troublesome noise on reflection seismograms.

In the present paper, we are interested in the theory of the reflection of a plane compressional incident wave at the free surface. In this theory a cubic expression occurs which also occurs in the theory of the Rayleigh waves. Furthermore our interest is primarily in obtaining numerical results, so that examples may be readily pictured. The results are primarily of theoretical interest since our waves are never plane and the medium is only rarely approximately homogeneous.

2. Reflection at the free surface. This problem originally treated by $\mathrm{Knott}^{2}$ and Zoeppritz ${ }^{3}$ leads to the relation

$$
\frac{R}{I}=\frac{v^{2} \sin 2 r_{1} \sin 2 i-V^{2} \cos ^{2} 2 r_{1}}{v^{2} \sin 2 r_{1} \sin 2 i+V^{2} \cos ^{2} 2 r_{1}},
$$

where $R, I, r_{1}, i, v$, and $V$ are respectively the amplitude of the reflected compressional wave, the amplitude of the incident compressional wave, the reflection angle of the shear wave, the angle of incidence, the velocity of the shear wave, and the velocity of the compressional wave.

We may make the substitutions $w=\sin ^{2} r_{1}=p^{2} v^{2}$ and $s=\lambda / \mu=2 \sigma /(1-2 \sigma)$ and obtain

$$
\begin{aligned}
\frac{R}{I} & =\frac{4 w(1-w)^{1 / 2}[1-(s+2) w]^{1 / 2}-(s+2)^{1 / 2}(1-2 w)^{2}}{4 w(1-w)^{1 / 2}[1-(s+2) w]^{1 / 2}+(s+2)^{1 / 2}(1-2 w)^{2}} \\
& =\frac{N(s, w)}{D(s, w)}=\frac{N^{2}}{N D}=\frac{N D}{D^{2}} .
\end{aligned}
$$

Now real values of both $N(s, w)$ and $D(s, w)$ are graphed on Fig. 1, for various values

* Received Dec. 2, 1944.

${ }^{1}$ Lord Rayleigh, On waves propagated along the plane surface of an elastic solid, Proc. Lond. Math. Soc. 17, 4-11 (1887).

2 C. G. Knott, Reflection and refraction of elastic waves, Phil. Mag. (5) 48, 64-97 (1899).

${ }^{3} \mathrm{~K}$. Zoeppritz, Über Erdbebenwellen VIIB, Göttinger Nachrichten 1919, 66-84. 
of $s$ or $\sigma$. It is seen that the graphs break up into two sets, one for $w \leqq 1 /(s+2)$ and the other for $w \geqq 1$. When $w=1 /(s+2)$ or $w=1$, the graphs of $N$ and $D$ both have vertical tangents. It will be observed that $N$ has two or no real zeros between 0 and $1 /(s+2)$ while $D$ has one real zero $\geqq 1.09574$. The double zero corresponds to

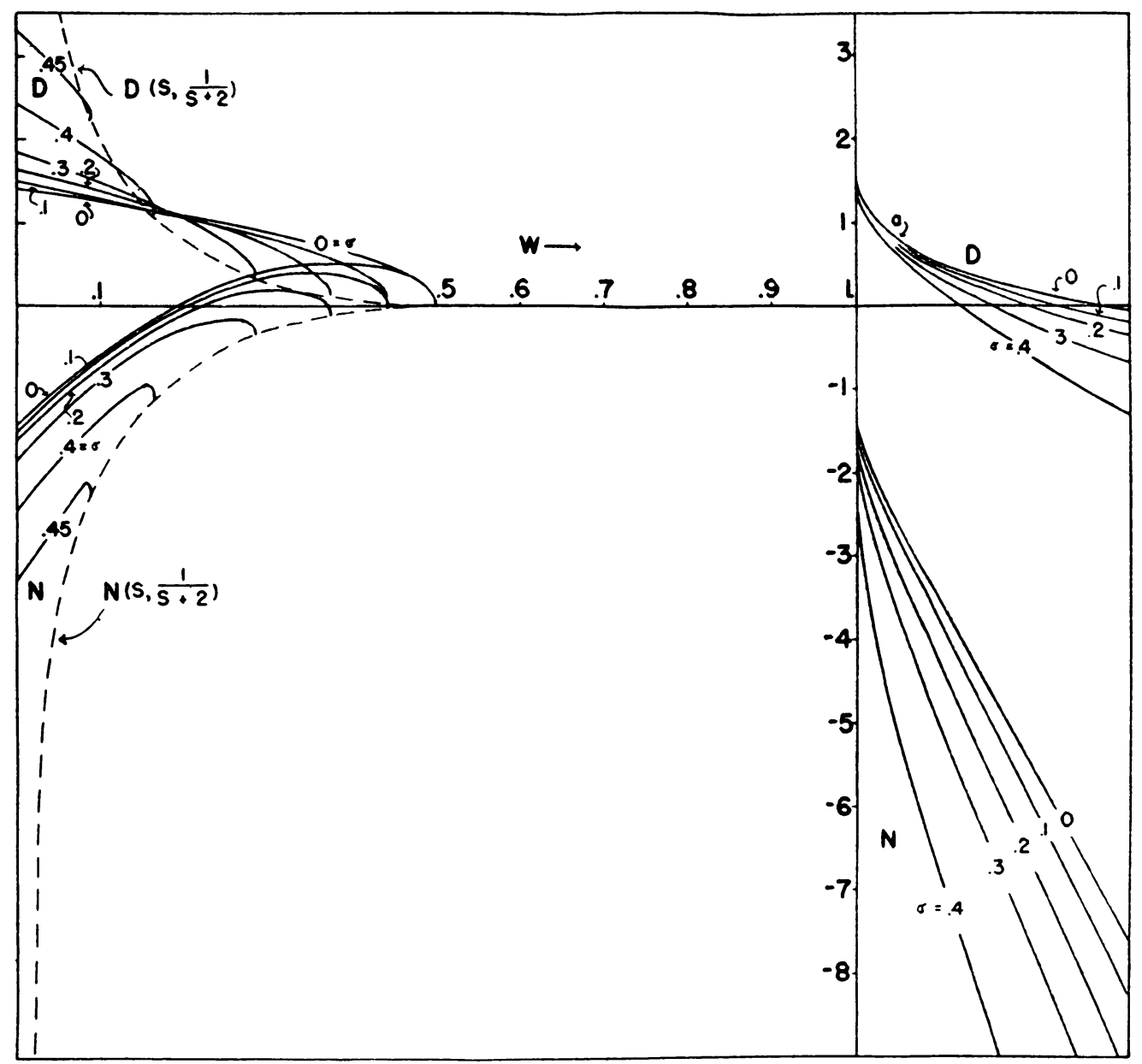

FIG. 1.

$\sigma=0.26308207, w=0.27969015$. For large values of $w$ we have $D \sim-2(s+1)(s+2)^{-1 / 2} w$ and $N \sim-8(s+2)^{1 / 2} w^{2}$. Hence the real zeros for $w>0$ are fully accounted for.

There is some interest in the plot of $R / I$ against $i$ for various $\sigma$ 's. This is shown in Fig. 2. Observe that the tangent to the curves at $i=0^{\circ}$ is horizontal and is vertical at $i=90^{\circ}$. Observe also that a discontinuity exists for $\sigma=0$ at $i=90^{\circ}$ since $\lim _{i \rightarrow 90^{\circ}}(R / I)_{\sigma=0}=+1$ and $(R / I)_{i=90^{\circ}, 0<\sigma<1 / 2}=-1$. One may note that the zeros of $(R / I)$ correspond to $i$ 's, for the given $\sigma$, for which there is no reflection of energy in the wave of compressional type. The change of sign of $(R / I)$ corresponds to a phase reversal. 


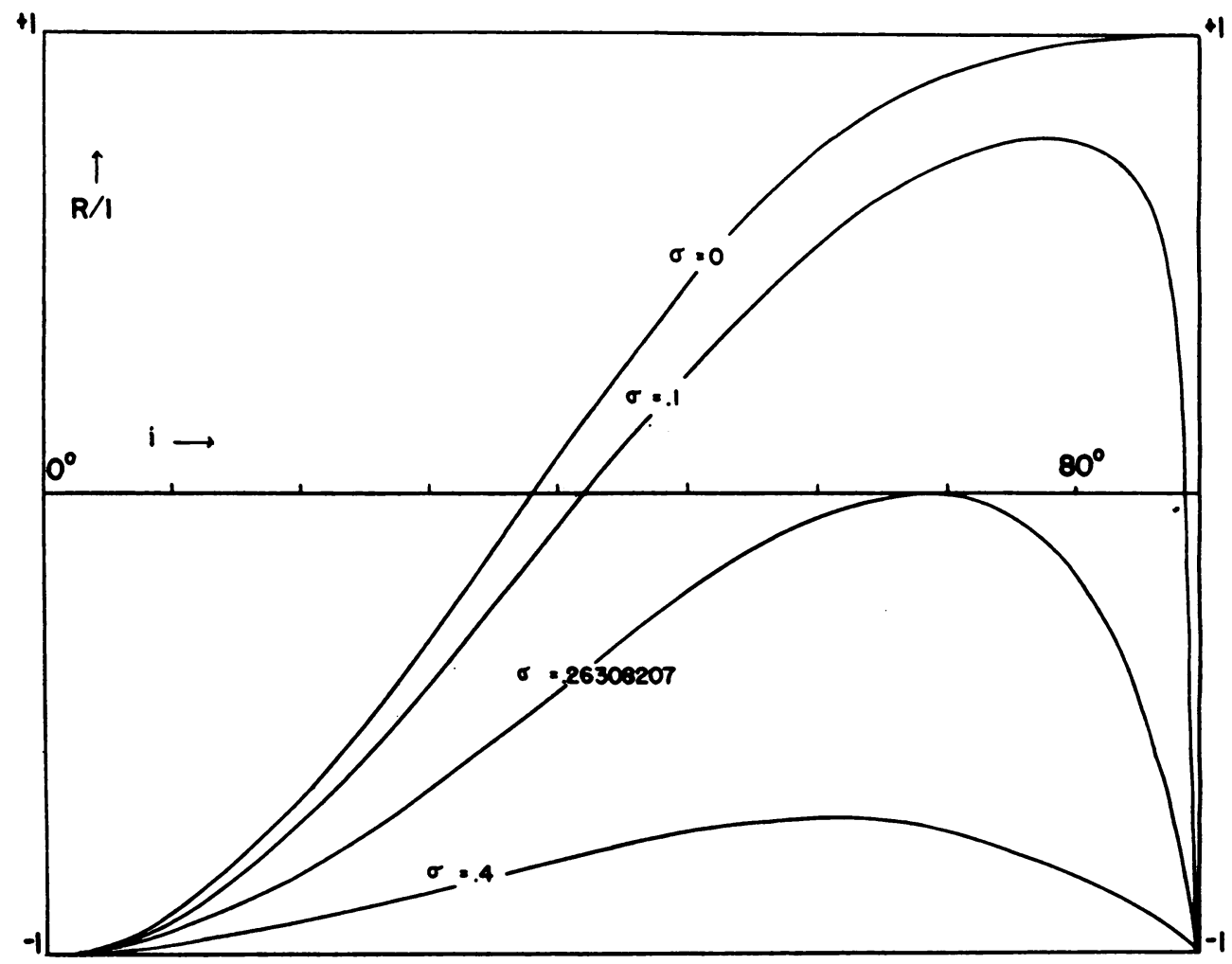

FIG. 2.

Since the zeros of $N(s, w)$ are of considerable interest we have plotted $i_{0}$ against $\sigma$ (where $i_{0}$ is incidence angle for which $N=R=0$ ) in Fig. 3. This gräph brings out a point which is indeed curious, namely if $\sigma=0.15$ then for $i_{0}=87.76^{\circ}$ all the reflected energy appears in the shear wave, whereas, if we add only $2.24^{\circ}$ to $i$ all the reflected energy appears in the compressional wave. Birch 4 records Poisson ratios for granite blocks of $0.093,0.096,0.116,0.086$ and 0.109 . These are selected low values. It is established in a later paragraph that $\left(d i_{0} / d \sigma\right)_{\sigma=0}=-\infty$ for the upper branch and $\left(d i_{0} / d \sigma\right)_{\sigma=0}=+0.178$ for the lower branch and $\left(d i_{0} / d \sigma\right)_{\sigma=0.263}=\infty$ for the upper and lower branches.

4 Francis Birch, Handbook of physical constants, Special Paper no. 36, Geol. Soc. of Amer., pp. 73-74 (1942).

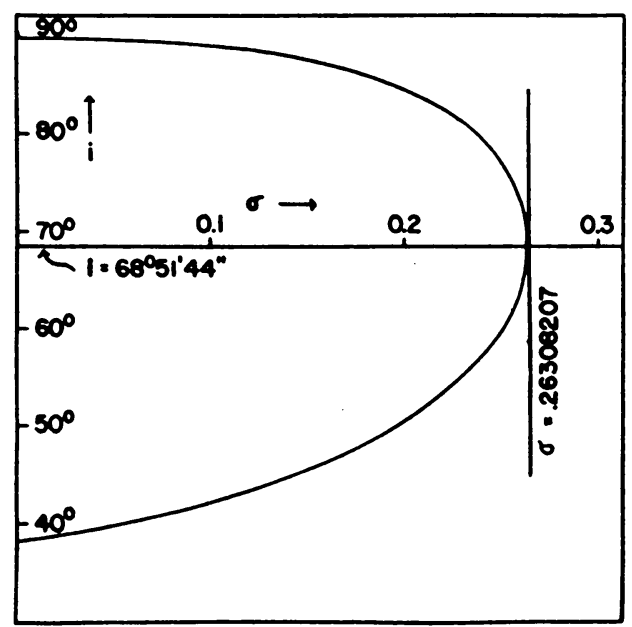

Fig. 3. 
If we rationalize either the denominator or numerator of (1) as indicated we obtain

$$
y \equiv N D \equiv 16(s+1) w^{3}-8(3 s+4) w^{2}+8(s+2) w-(s+2) .
$$

The curves for $y=y(s, w)$ are plotted in Fig. 4. The fixed point at $w=1.0957444$,

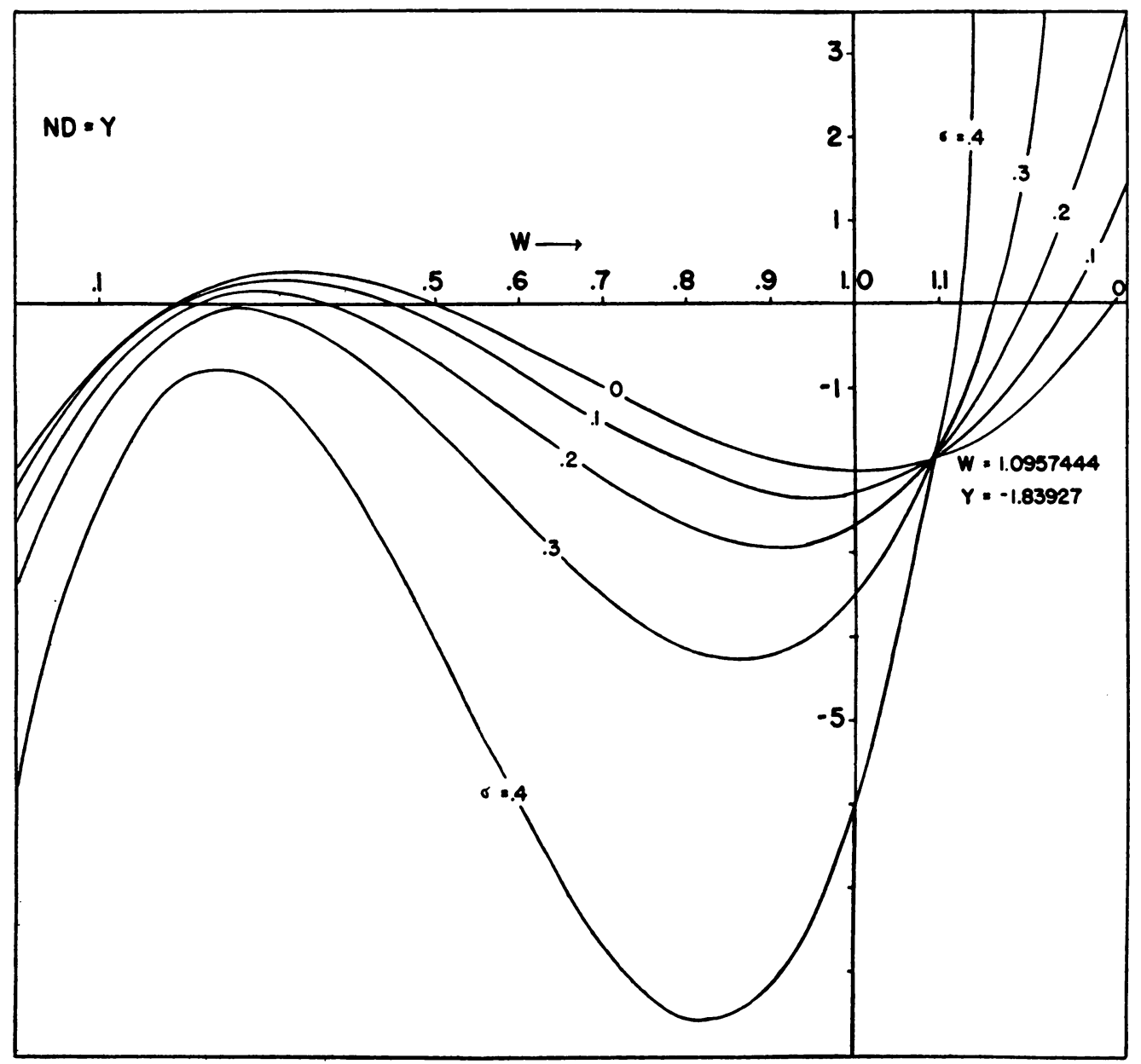

FIG. 4.

$y=-1.83927$ will be noted. It corresponds to the $w$ for which $\partial y / \partial s=16 w^{3}-24 w^{2}$ $+8 w-1=0$. Note also that

$$
y\left(s_{1}, w\right)-y\left(s_{2}, w\right)=\left(s_{1}-s_{2}\right)(\partial y / \partial s) .
$$

If either $N$ or $D$ is zero then $y$ is zero. Assume $y=0$ while $s$ and $w$ vary. Then after a few reductions we obtain

$$
\frac{d i_{0}}{d \sigma}=-2 \frac{(s+1)^{2}(s+2)}{\sin 2 i_{0}} \cdot \frac{(\partial y / \partial s)}{(\partial y / \partial w)_{w-w_{0}}} .
$$


This relationship should be kept in mind in connection with the plot of Fig. 2. Note that the double zero of the cubic (2) is the common zero of $y=0$ and $\partial y / \partial w=0$ (yielding after elimination of $w$ the relation $33 s^{3}+12 s^{2}-27 s-30=0$, so that $s_{0}=1.11043541$ and $\sigma=0.26308207$ while $i_{0}=68^{\circ} 51^{\prime} 44^{\prime \prime} ;^{5}$ hence the $\partial y / \partial w$ in (4) is zero, leading to the vertical tangent. Note that the point $\sigma=0, i_{0}=90^{\circ}$ is not attained on Fig. 3 but

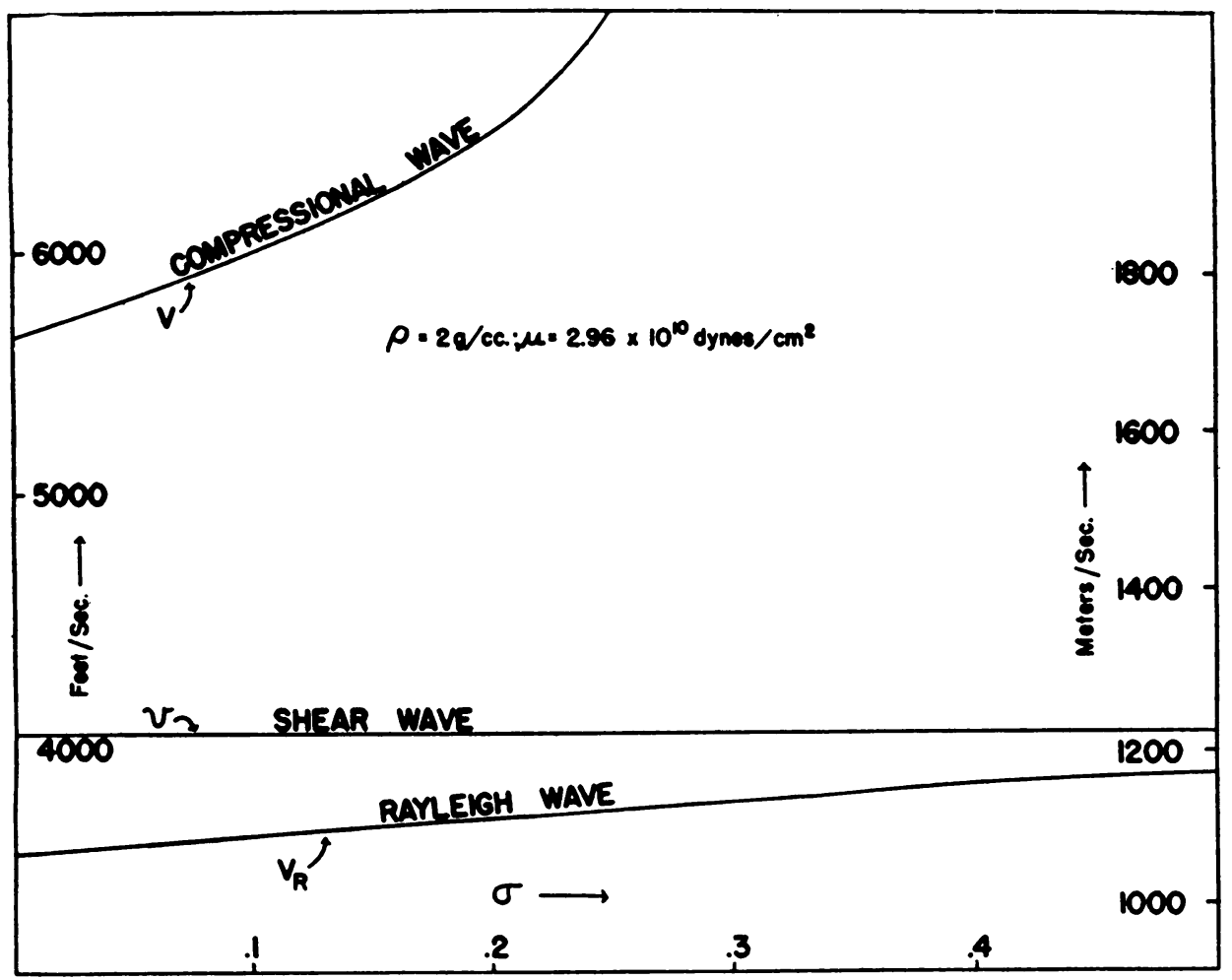

Fig. 5.

that as we approach this point from $\sigma>0, d i_{0} / d \sigma \rightarrow-\infty$ because $\sin 2 i_{0} \rightarrow 0$ whereas the other factors are bounded away from zero.

3. Rayleigh waves. In the usual theory of Rayleigh waves ${ }^{6}$ a cubic equation occurs which, using our s-notation, can be written in the form,

$$
16(s+1)-8(3 s+4) \bar{w}+8(s+2) \bar{w}^{2}-(s+2) \bar{w}^{3}=0,
$$

where $\bar{w}=V_{R}^{2} / v^{2}, v$ and $V_{R}$ being respectively the velocities of the shear wave and Rayleigh wave. Thus when $y=0, w=\bar{w}^{-1}$. But $w=\sin ^{2} r_{1}=p^{2} v^{2}$ where $p=\sin i / V$ $=\sin r_{1} / v$. Hence $p^{2} v^{2}=v^{2} / V_{R}^{2}$ so $p=1 / V_{R}$. Thus $p$, which for real values of $i$ or $r_{1}$, can be interpreted in terms of the reciprocal of the velocity with which the wave

5 B. Gutenberg, Energy ratio of reflected and refracted seismic waves, Bull. Seis. Soc. Amer. 34, 85-102 (1944).

'J. B. Macelwane, Theoretical seismology, Part I, Wiley \& Sons, New York, 1936, p. 114, Eq. (5.33). 


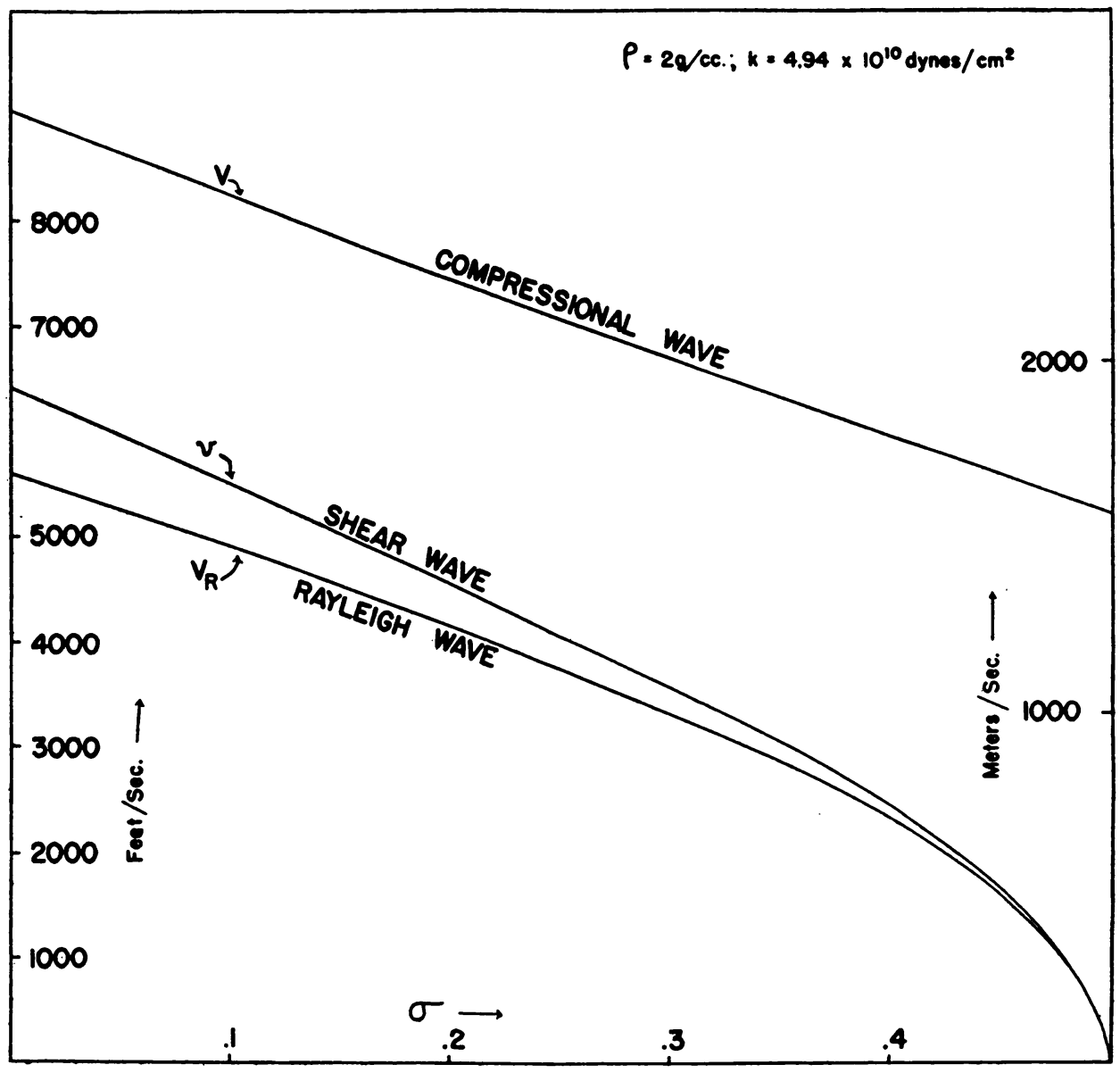

Fig. 6.

sweeps along the surface, $\partial t / \partial x$, has the same interpretation in the case of the Rayleigh wave.

The Rayleigh wave case corresponds to the zero of $D(s, w)$ which in turn yields a poristic problem when $I \equiv 0 .^{7}$

The Rayleigh wave velocities can be readily computed by solving the cubic equation or by an inspection of Fig. 4. However, Figs. 5 and 6 show $V$, v, and $V_{R}$ for the respective cases where $\mu=$ constant $=2.96 \times 10^{10}$ dynes $/ \mathrm{cm} .{ }^{2}$, and where $k=(\mu / 3)(3 s+2)=$ constant $=4.94 \times 10^{10}$ dynes $/ \mathrm{cm}^{2}=$ modulus of compression for various values of $\sigma$.

${ }^{7}$ T. Sakai, On the propagation of tremors over plane surface, Geophysical Magazine, Tokyo, 8, 1-71 (1934). 